\title{
Water Disinfection Byproducts Increase Natural Transformation Rates of Environmental DNA in Acinetobacter baylyi ADP1
}

\author{
David Mantilla-Calderon, ${ }^{\dagger}$ Michael J. Plewa, ${ }^{\S}, \|_{\odot}$ Grégoire Michoud, $^{\ddagger}$ Stelios Fodelianakis, ${ }^{\ddagger}$ \\ Daniele Daffonchio, ${ }^{\ddagger}$ and Pei-Ying Hong* ${ }^{+\dagger}$
} ${ }^{\dagger}$ Water Desalination and Reuse Center, Division of Biological and Environmental Science and Engineering, and ${ }^{\star}$ Division of
Biological and Environmental Science and Engineering, King Abdullah University of Science and Technology (KAUST), Thuwal
23955-6900, Saudi Arabia

${ }^{\S}$ Department of Crop Sciences, and "Safe Global Water Institute, University of Illinois at Urbana-Champaign, Urbana, Illinois 61801, United States

Supporting Information

\begin{abstract}
The process of natural transformation allows for the stable uptake, integration, and functional expression of extracellular DNA. This mechanism of horizontal gene transfer has been widely linked to the acquisition of antibiotic resistance and virulence factors. Here, we demonstrate that bromoacetic acid (BAA) - a regulated drinking water disinfection byproduct (DBP) - can stimulate natural transformation rates in the model organism Acinetobacter baylyi ADP1. We demonstrate that transformation stimulation in response to BAA is concentration-dependent and is linked to the ability of this compound to generate DNA damage via oxidative stress. In presence of BAA, transcription of $\operatorname{rec} A$ was upregulated $20-40 \%$ compared to the nontreated controls, indicating that this component of the DNA damage response could be associated with the increase in transformation. Other genes associated with DNA translocation across the cytoplasmic membrane (i.e., pilX, comA) did not exhibit increased transcription in the presence of BAA, indicating that the enhancement of transformation is not associated with increased translocation rates of environmental DNA. Overall, these results lead us to speculate that elevated recA transcription levels could lead to increased integration rates of foreign DNA within the recipient cell during DNA repair. Lastly, we show that an artificial DBP cocktail simulating the environmental concentrations of five water DBP classes stimulates natural transformation by almost 2-fold. The results of this study suggest that mutagens like DBPs may play an important role in enhancing the fixation rates of extracellular DNA in the environmental metagenome.
\end{abstract}

\section{INTRODUCTION}

The emergence and rapid spread of antimicrobial resistance (AMR) among bacterial pathogens is a serious public health concern. ${ }^{1}$ Traditionally, clinical settings were considered the main site for the evolution of AMR. However, over the past decade, antibiotic resistance genes (ARGs) conferring resistance to last-resort antibiotics have been detected in multiple environmental compartments, including agricultural soils, ${ }^{2}$ sediments, ${ }^{3,4}$ and surface waters. ${ }^{4-6}$ Consequently, AMR is no longer considered to be a phenomenon exclusive to clinical settings ${ }^{2,7}$ but, rather, one that is also prevalent in the environment.

Wastewater discharges constitute a key source of environmental resistance, as they carry high concentrations of emerging microbial pollutants (e.g., ARGs and antibiotic resistant bacteria, ARB). ${ }^{3,8,9}$ To mitigate this concern, most countries treat wastewater through a series of physical, biological, and chemical processes, including a final disinfection step, to decrease the nutrient and bacterial counts to permissible levels. The processing of municipal and agricultural wastewaters to kill pathogens is important to protect public health and the environment. However, it had been demonstrated earlier that different disinfectants or disinfection processes alter the toxicological characteristics of treated wastewaters. ${ }^{10-14}$ Even with disinfection, studies reported increases in the occurrence of ARB among various types of bacterial taxa (e.g. Enterobacteriaceae, Aeromonas spp., Acinetobacter spp.) in downstream receiving waters. ${ }^{15-17}$ Besides direct deposition of microbial contaminants, horizontal flow of ARGs between wastewater-borne bacteria and the native microbial communities could potentially explain the increased prevalence of ARB in wastewater-impacted environments. ${ }^{16,18}$ The contribution of horizontal gene transfer (HGT) to the ARB enrichment phenomenon is inferred from (i) the observation that the resistome fraction comprising ARGs of clinical relevance-in wastewater and the environment-are predominantly associated with mobile genetic elements ${ }^{2,19}$ and (ii) the capacity of

Received: February 1, 2019

Revised: April 22, 2019

Accepted: May 3, 2019

Published: May 3, 2019 
sublethal doses of disinfectants (e.g., chlorine) to stimulate conjugation rates by $2-5$-fold. ${ }^{20}$ To the best of our knowledge, environmental studies had focused exclusively on the contribution of conjugation in the spread of AMR. ${ }^{21}$ However, this mechanism of HGT does not present a comprehensive picture of the environmental fate and persistence of ARGs, especially those that exist as free extracellular DNA.

In addition to the effect of chlorine on conjugation, many disinfection byproducts (DBPs), which are an unintended consequence of water disinfection, are genotoxins and mutagens in a number of biological systems. ${ }^{22,23}$ In bacteria, exposure to mutagens lead to the transcriptional activation of the $r e c A$ gene as part of DNA damage response pathways. ${ }^{24,25}$ The product of $r e c A$ is involved in the initiation of homologous recombination, a fundamental process for both repair of DNA double-strand breaks $^{26}$ and chromosomal integration of foreign DNA during natural transformation. ${ }^{27}$

On the basis of the previous observations, we hypothesized that environmental stressors, such as DBPs arising from the final disinfection step of most wastewater treatment plants, can trigger DNA repair in naturally competent bacteria. This would facilitate the integration process of foreign DNA during natural transformation and, hence, allow microorganisms to gain new functional traits. The objective of this study was to determine whether exposure to such environmental mutagens could enhance the process of transformation in naturally competent bacteria. As DBPs are particularly abundant in treated wastewater effluents, ${ }^{28,29}$ we tested our hypothesis using the interaction between bromoacetic acid (BAA), a mutagenic, genotoxic, and regulated $\mathrm{DBP},{ }^{30,31}$ and the ubiquitous and naturally competent bacterium Acinetobacter baylyi ADP1.

\section{MATERIAL AND METHODS}

2.1. Bacterial Strains and Culture Conditions. A reporter strain of A. baylyi ADP1 (BD413) was used in this study. ${ }^{32}$ After a successful natural transformation event, the reporter strain expresses spectinomycin resistance and GFP+ phenotype. ${ }^{32} A$. baylyi was routinely cultivated at $37^{\circ} \mathrm{C}$ in LB Miller broth and agar, supplemented with kanamycin $\left(50 \mu \mathrm{g} \mathrm{mL} \mathrm{m}^{-1}\right)$ and rifampicin $\left(50 \mu \mathrm{g} \mathrm{mL}^{-1}\right)$. Salmonella enterica serovar Typhimurium TA100 was propagated in LB Miller broth supplemented with ampicillin at a final concentration of $25 \mu \mathrm{g} \mathrm{mL}^{-1}$.

2.2. Mutagenicity Test. The mutagenic potential of BAA was evaluated by a modified Ames preincubation reversion assay in the tester strain S. enterica serovar Typhimurium TA100, as previously described. ${ }^{33}$ S. enterica serovar Typhimurium TA100 was used for mutagenicity tests as this assay is considered the gold standard for assessing mutagenicity in bacterial systems. ${ }^{34}$ Briefly, a standardized suspension of $2 \times 10^{9}$ cells $\mathrm{mL}^{-1}$ of the tester strain was incubated at $37^{\circ} \mathrm{C}$ with $0-600 \mu \mathrm{MBAA}$ for $1 \mathrm{~h}$. Subsequently, $5 \times 10^{8}$ cells were plated in Vogel-Bonner (VB) minimal medium, and histidine revertant colonies were enumerated after $72 \mathrm{~h}$ of incubation at $37{ }^{\circ} \mathrm{C}$. BAA and glutathione $(\mathrm{GSH})$ coincubation assays were set to contain an equimolar GSH concentration relative to BAA. Incubation and sample processing were done according to standard methods.

2.3. Concentration-Response Transformation Stimulation Assays. A. baylyi was inoculated in $50 \mathrm{~mL}$ of LB Miller broth supplemented with kanamycin $\left(50 \mu \mathrm{g} \mathrm{mL}{ }^{-1}\right)$ and rifampicin $\left(50 \mu \mathrm{g} \mathrm{mL}^{-1}\right)$ and incubated overnight at $37{ }^{\circ} \mathrm{C}$ with stirring $(200 \mathrm{rpm})$. Subsequently, $A$. baylyi cell cultures were centrifuged $\left(4{ }^{\circ} \mathrm{C}, 6500 \mathrm{rpm}\right.$ for $\left.10 \mathrm{~min}\right)$ and washed twice with cold phosphate buffer (PPB). Cell pellets were suspended in $1 \mathrm{~mL}$ of $\mathrm{PPB}$ and adjusted to a standardized suspension containing $2 \times 10^{10}$ cells $\mathrm{mL}^{-1}$. One hundred microliters of standardized suspension was added to fresh LB Miller broth (without antibiotics) containing $2 \mu \mathrm{g} \mathrm{mL}^{-1}$ of donor DNA and $0-600 \mu \mathrm{M}$ BAA (initial inoculum of $2 \times 10^{9}$ cells $\mathrm{mL}^{-1}$ and 1 $\mathrm{mL}$ of final volume per assay tube). Since natural transformation events occur at extremely low frequencies, ranging from $10^{-5}$ to $10^{-8}$, it was important to optimize the detection limit of our assays by utilizing high concentrations of donor DNA and high densities of A. baylyi cells. Assays were incubated for $24 \mathrm{~h}$ at 37 ${ }^{\circ} \mathrm{C}$ with stirring (200 rpm). To determine baseline mutation rates that could spontaneously lead to the expression of the cassette, a series of controls lacking the donor DNA were set up in parallel in the $0-600 \mu \mathrm{M}$ BAA range. These controls include (i) A. baylyi cells alone (no BAA, no donor DNA added) and (ii) A. baylyi cells + BAA $(0-600 \mu \mathrm{M}$, no donor DNA added). No transformants were detected in both types of controls in all biological replicates. This suggests that BAA-induced mutations without the presence of any donor DNA would not result in the expression of spectinomycin resistance or GFP in the reporter strain. Neither would A. baylyi undergo spontaneous mutation that would lead to the expression of the aadA::GFP cassette in the absence of stressor (i.e., BAA).

Donor DNA was generated by PCR using the primer pair 5' ATT ATT GAA TTC GGT AGA GCC GTT TAT GAA-3' forward and 5'-TTT GCC CAC TAC CTT GGT GAT-3' reverse, ${ }^{32}$ utilizing as template a cloning vector $(\mathrm{pCLT})$ that contains the functional promoter $\left(P_{r r n}\right)$ that activates the aadA::GFP transcriptional fusion. The pCLT cloning vector is propagated in Escherichia coli K12. This primer set amplifies the rescue cassette $r b c L-P_{r r n}$-aadA, which inserts by homologous recombination upstream the silenced aadA::GFP fusion. The resulting $2.1 \mathrm{~kb}$ amplicon containing the $r b c L-P_{r r n}$-aadA fragment was purified using the Wizard SV Gel and PCR Clean-Up System (Promega), and purified DNA was quantified with the Qubit DNA BR Assay Kit (Invitrogen). More details on the transformation reporter system were described in an earlier study by Rizzi et al. ${ }^{32}$

After $24 \mathrm{~h}$, total cell counts were determined by plating in LB Miller agar supplemented with kanamycin $\left(50 \mu \mathrm{g} \mathrm{mL}^{-1}\right)$ and rifampicin $\left(50 \mu \mathrm{g} \mathrm{mL}^{-1}\right)$ and incubated for $24 \mathrm{~h}$ at $37{ }^{\circ} \mathrm{C}$. Screening for transformants was first achieved by additionally supplementing LB Miller plates with spectinomycin (100 $\mu \mathrm{g}$ $\left.\mathrm{mL}^{-1}\right)$. No colonies resistant to spectinomycin were detected at any BAA concentration when donor DNA was not present, suggesting the lack of spontaneous expression of gene cassettes by $A$. baylyi in the absence of donor DNA. In the assays containing the donor DNA, insertion of the donor was further confirmed by colony PCR in five randomly selected transformant colonies per plate. Colony PCR was done using the primer pair 5'-ATC TTT CTA TTG TTG TCT TGG AT-3' forward and 5'-GGT CAC CGT AAC CAG CAA ATC AA-3' reverse. $^{32}$ This primer pair anneals downstream and upstream of the deleted promoter. In the presence of the promoter, introduced after a successful transformation of the donor DNA, the PCR amplicon would be $333 \mathrm{bp}$, whereas an unsuccessful transformation would derive a PCR amplicon of $190 \mathrm{bp}$. Therefore, the amplicon size would allow for differentiation between cells with and without the donor DNA insertion. All screened colonies were tested positive for the donor DNA insertion, confirming that the observed phenotype corresponds to the insertion of the donor DNA and not to 
Assays with Salmonella TA 100

a

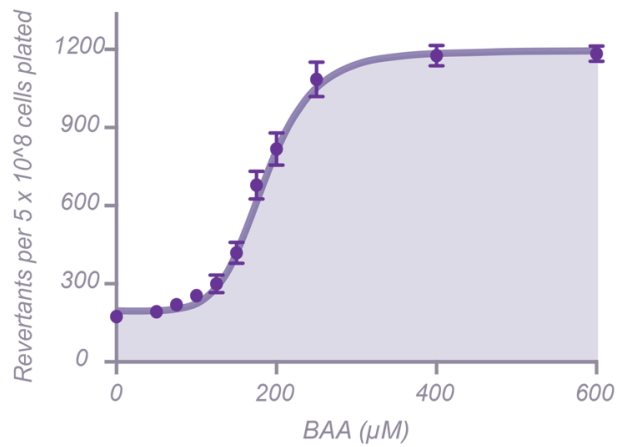

c

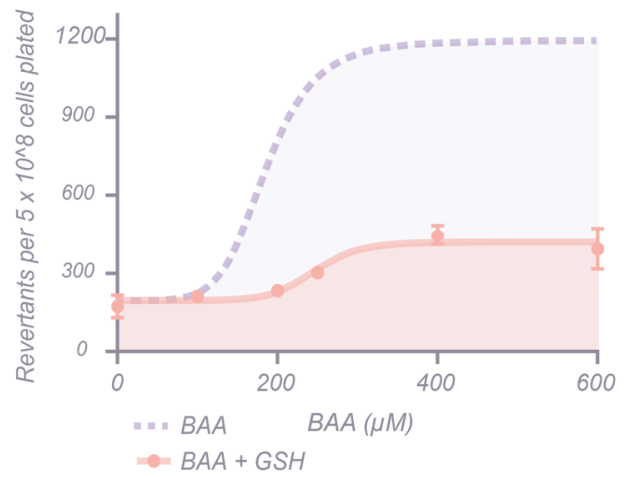

Assays with Acinetobacter baylyi ADP1 reporter strain .

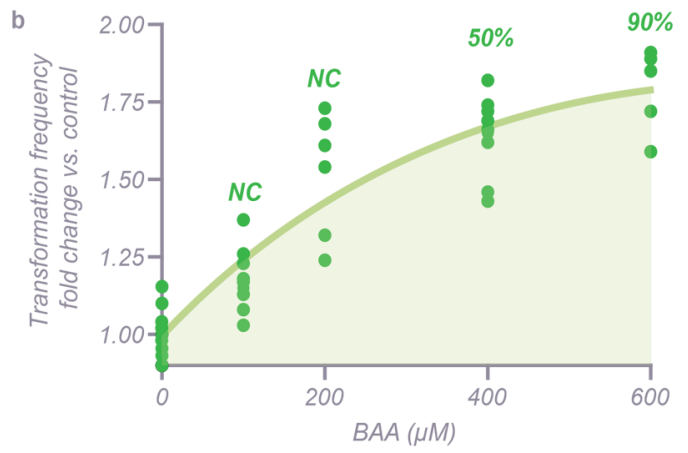

d
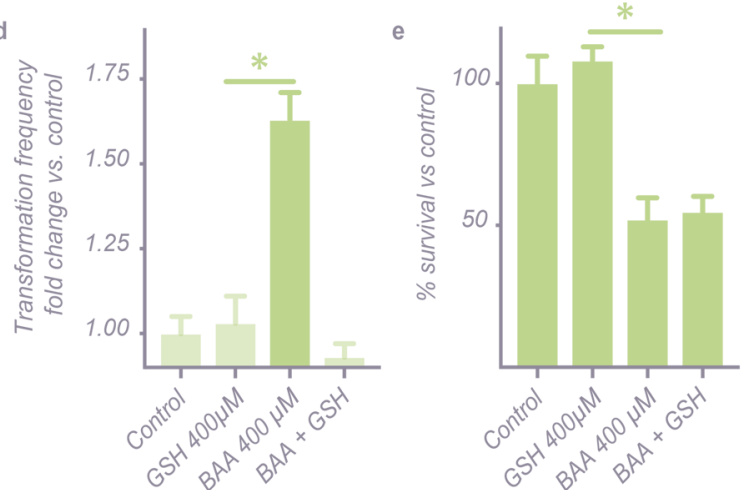

Figure 1. BAA enhances transformation rates in A. baylyi in a ROS-dependent manner. (a) S. enterica serovar Typhimurium TA100 reversion assay showing the mutagenicity of BAA. Data were from four independent replicates. (b) Transformation frequencies of A. baylyi ADP1 exposed to 0-600 $\mu \mathrm{M}$ BAA. Data is reported as fold-change transformation frequency vs control. Green circles correspond to individual fold-change values of independent biological replicates at each BAA concentration. An ANOVA test statistic demonstrated a significant $F$-test response $(F=74.09 ; P<$ $0.001)$. Responses in all treatment concentrations were highly significant over the $0 \mu \mathrm{M} \mathrm{BAA}$ control $(P<0.001)$. Power of performed test with $\alpha=$ 0.050:1.000. NC and percentages above data points at each specified BAA concentration depict the percentage of $A$. baylyi ADP1 cell death at that particular BAA concentration; NC = noncytotoxic. (c) Mutagenic response in TA100 to BAA + glutathione (GSH) treatments. GSH and BAA were added at equimolar concentrations $(n=4)$. (d) Fold-change transformation frequencies of $A$. baylyi ADP1 exposed to BAA and BAA + GSH $(n=3)$. (e) Percentage of A. baylyi ADP1 cell survival in BAA- and BAA + GSH-treated cell cultures $(n=3)$. Plots indicate the mean response \pm SE. Statistical comparisons in bar graphs were done using ANOVA all pairwise comparisons. * denotes $P<0.001$. Power of the performed test with $\alpha=0.050: 1.000$.

spontaneous mutations that could lead to the expression of the cassette.

Transformation frequency was defined as the ratio of transformants over the total number of cells in each assay vial. Fold induction was defined as the ratio of the transformation frequency of each treatment, over the average transformation frequency for the $0 \mu \mathrm{M}$ BAA control in the same replicate run. Statistical differences between treatments were calculated by ANOVA. The power test was calculated using Sigma Plot v13 (Systat Software Inc., San Jose, CA).

2.4. BAA and Glutathione Coincubation Assays. A BAA and glutathione (GSH) coincubation assay was designed to determine whether reactive oxygen species (ROS) are involved in the increased transformation and cytotoxic response to BAA exhibited by $A$. baylyi. First, the ability of glutathione to modulate mutagenicity was tested in $S$. enterica serovar Typhimurium TA100. For this, the procedure described in section 2.2 was followed with a slight modification. Besides the addition of BAA, an equimolar concentration of GSH was added to the same assay tube (i.e., $400 \mu \mathrm{M} \mathrm{BAA}+400 \mu \mathrm{M} \mathrm{GSH}$ ). Assay incubation and quantification of revertants were then conducted as described in section 2.2.

Once it was demonstrated that an equimolar concentration of GSH inhibits the mutagenic output of BAA in TA100, transformation assays in A. baylyi were repeated in the presence of equimolar concentrations of GSH (i.e., $400 \mu \mathrm{M}$ BAA +400 $\mu \mathrm{M}$ GSH). Assay incubation, transformation frequencies, and cytotoxic response were determined as described in section 2.3.

2.5. Biomass Collection for BAA Toxico-Genomics and RNA Extraction. BAA cytotoxicity was detected at concentrations higher than $200 \mu \mathrm{M}$ [Figure S1, Supporting Information (SI)]. Given that the transformation stimulation phenotype is linked to mutagenic potential of BAA and not to its cytotoxic output, transcriptome analysis was restricted to the $0-200 \mu \mathrm{M}$ concentration range, where no increased cytotoxicity was detected (Figures S1 and S2, SI). This is to avoid capturing the cytotoxic response to BAA, which is not accounting for the observed increase in natural transformation.

One hundred microliters of an overnight culture of $A$. baylyi was inoculated in $100 \mathrm{~mL}$ of fresh LB Miller broth and incubated at $37{ }^{\circ} \mathrm{C}$ with stirring $(200 \mathrm{rpm})$. After reaching an $\mathrm{OD}_{660}$ of 0.1 , cell cultures were spiked with $2 \mu \mathrm{g} \mathrm{mL}^{-1}$ of donor DNA and 100 , 150 , and $200 \mu \mathrm{M}$ BAA, or mock spiked with an equal volume of carrier (DMSO) for the $0 \mu \mathrm{M}$ control. Cell cultures were placed back in incubation, and $10 \mathrm{~mL}$ samples were taken at $0,10,30$, and $60 \mathrm{~min}$. After collection, samples were immediately chilled in an ethanol ice bath and cell pellets collected by centrifugation at $7000 \mathrm{rpm}$ for $15 \mathrm{~min}$ at $4{ }^{\circ} \mathrm{C}$. Cell pellets were subsequently 
suspended in RNAlater stabilization solution (Thermo Scientific), following manufacturer instructions. Cell pellets were long-term stored at $-80{ }^{\circ} \mathrm{C}$.

Cell pellets were extracted for total RNA with an automated Maxwell RSC Instrument, operating with a Maxwell RSC SimplyRNA Cell Kit (Promega). RNA extraction was done following the manufacturer instructions with a minor technical modification. In order to achieve complete DNA digestion during extraction, 7 units extra of DNase I per sample was added to the DNA digestion step. RNA integrity was determined using a 2200 TapeStation System (Agilent), whereas RNA concentration was assessed with the Qubit RNA HS Assay Kit (Invitrogen).

2.6. RNA-seq and Data Analysis. RNA samples were sent to the KAUST Bioscience Core Lab for paired-end sequencing on an Illumina HiSeq 4000 platform. Libraries were first constructed using a Tru Seq Stranded Total RNA Kit. The quality of the reads was assessed using FastQC software, ${ }^{35}$ the Illumina primers were then removed with the Trimmomatic program. ${ }^{36}$ The abundances of transcripts were quantified with kallisto software, ${ }^{37}$ and the differential analyses were done with the sleuth program. ${ }^{38}$ The genome of Acinetobacter sp. ADP1 (assembly ASM4684v1) was used as reference for kallisto. Concentration-response behavior was evaluated by testing for differences at the gene expression (transcripts per million, TPM) with increasing concentration of BAA, irrespective of time. For that, we compared a full model (TPM $\sim \mathrm{BAA}+$ time) to a reduced model (TPM $\sim$ time) in sleuth. All transcriptomic FASTQ files are deposited in the European Nucleotide Archive under study accession number PRJEB28698.

2.7. Reverse Transcription-Quantitative Polymerase Chain Reaction (RT-qPCR) Quantification. Transcriptional changes were confirmed by RT-qPCR using the $t=60 \mathrm{~min}$ samples from the toxico-genomic experiment. First, cDNA was generated priming with random hexamers using the SuperScript III First Strand cDNA Synthesis Kit (Thermo Fisher Scientific) following manufacturer's instructions. Copy numbers were determined by absolute quantification as previously described ${ }^{39}$ using the primers listed in Table S1 (SI).

2.8. DBP Cocktail Transformation Stimulation Assays. One hundred microliters of an overnight culture of A. baylyi was inoculated in $100 \mathrm{~mL}$ of fresh LB Miller broth and incubated at $37^{\circ} \mathrm{C}$ with stirring (200 rpm). After reaching an $\mathrm{OD}_{660}$ of 0.1 , aliquots of $1 \mathrm{~mL}$ were dosed to $20 \mathrm{~mL}$ glass vials containing a simplified DBP cocktail or an equal volume of DMSO and incubated for $1 \mathrm{~h}$ at $37{ }^{\circ} \mathrm{C}$ with stirring (200 rpm). A detailed description of the DBP cocktail can be found in the Supporting Information (Table S2). Transformation frequencies were determined by following the protocol described for the BAA concentration-response transformation stimulation assays.

2.9. Determination of Potential Interaction between BAA and Donor DNA. To confirm that the transformation induction phenotype is not caused by a potential direct BAADNA interaction, a separate set of experiments was conducted in which the donor DNA was first pre-exposed to BAA prior to transformation in the absence of BAA. First, donor DNA was pre-exposed to BAA $(0-600 \mu \mathrm{M})$ for $1 \mathrm{~h}$ and subsequently purified using ethanol precipitation. A $1 \mathrm{~h}$ exposure time was used as previous transformation assays had shown that $1 \mathrm{~h}$ of exposure to BAA was enough to enhance transformation (Figure S1c, SI). Transformation assays were performed as described in section 2.3, but this time BAA was not added in the culture media and pre-exposed DNA was used instead.

\section{RESULTS AND DISCUSSION}

3.1. BAA Stimulates Natural Transformation Rates in a Concentration-Response Manner. BAA-induced mutagenicity concentration response curves were first generated using a modified preincubation test of the his ${ }^{-}$reversion assay in $S$. enterica serovar Typhimurium strain TA100. ${ }^{30,40}$ TA100 expressed BAA-induced mutagenicity in the concentration range of $100-600 \mu \mathrm{M}$, with a linear response from 100 to 250 $\mu \mathrm{M}$ BAA (Figure 1a). Subsequently, we determined whether $\mathrm{BAA}$ at these mutagenic concentrations enhanced natural transformation by using a recipient-reporter strain derivative of A. baylyi ADP1 (BD413). ${ }^{32}$ This strain carries a markerreporter rescue cassette $(r b c L-\Delta \mathrm{P}$ aadA::GFP) integrated in the bacterial chromosome between the $\operatorname{lip} B$ and $\operatorname{lip} A$ genes. The transcriptional fusion ( $a a d A:: G F P)$, encoding for spectinomycin resistance $(a a d A)$ and the green fluorescent protein (GFP), lacks a functional promoter and therefore is not expressed in the recipient strain. A donor DNA carrying a constitutive functional promoter $\left(r b c L-P_{r r n}-a a d A\right)$ is used to restore the expression of aadA::GFP. Upon uptake of the donor DNA, homologous recombination takes place between the flanking $r b c L$ and $a a d A$ loci, leading to the insertion of the $P_{r r n}$ promoter upstream of the aadA::GFP fusion. The promoter integration activates the transcription of the gene fusion, restoring spectinomycin resistance and the fluorescent phenotype.

In accordance with the study hypothesis, BAA significantly increased the transformation frequencies of the reporter strain by nearly 2 -fold at the highest BAA concentration $(600 \mu \mathrm{M})$. Moreover, transformation stimulation followed a BAA concentration-response pattern (Figure $1 \mathrm{~b}$ ), establishing a direct link between BAA exposure and the increased rates of DNA transformation. It is important to highlight that no transformants were detected in the control assays lacking donor DNA and that insertion of donor DNA was further confirmed by PCR in the DNA-positive assays. These results demonstrate that the concentration-response relationship does not correspond to increased mutagenic rates that could lead to spontaneous expression of the cassette but, instead, results from an actual increase in transformation frequencies in the presence of BAA.

3.2. Natural Transformation Stimulation Is Triggered by DNA Damage via Oxidative Stress. Toxicity end points of haloacetic acids (HAAs) in TA100 included mutagenic as well as cytotoxic responses. Recent information demonstrated that the toxicity of the monohalogenated HAAs involves thiol/ thiolate reactivity to polypeptides and enzymes. ${ }^{41}$ The involvement of the induction of oxidative stress in monohalogenated HAAs genotoxicity was also previously demonstrated using reactive oxygen species (ROS) inhibitors and human toxico-genomic analyses. ${ }^{42-44}$ In addition, the results from these studies showed no direct interaction between monohalogenated HAAs and DNA. To confirm the absence of direct $\mathrm{BAA}-\mathrm{DNA}$ interaction, transformation assays (no BAA added in culture media) utilizing only donor DNA that was preexposed to increasing BAA concentrations $(0-600 \mu \mathrm{M})$ were performed. Pre-exposed donor DNA did not lead to increased natural transformation frequencies (Figure S3, SI), confirming that the observed transformation stimulation phenotype does not arise from direct BAA-DNA interactions.

To establish a direct link between ROS-mediated DNA damage and the transformation stimulation phenotype, the mutagenic response was modulated using an ROS scavenger. An experiment with the addition of equimolar concentrations of 


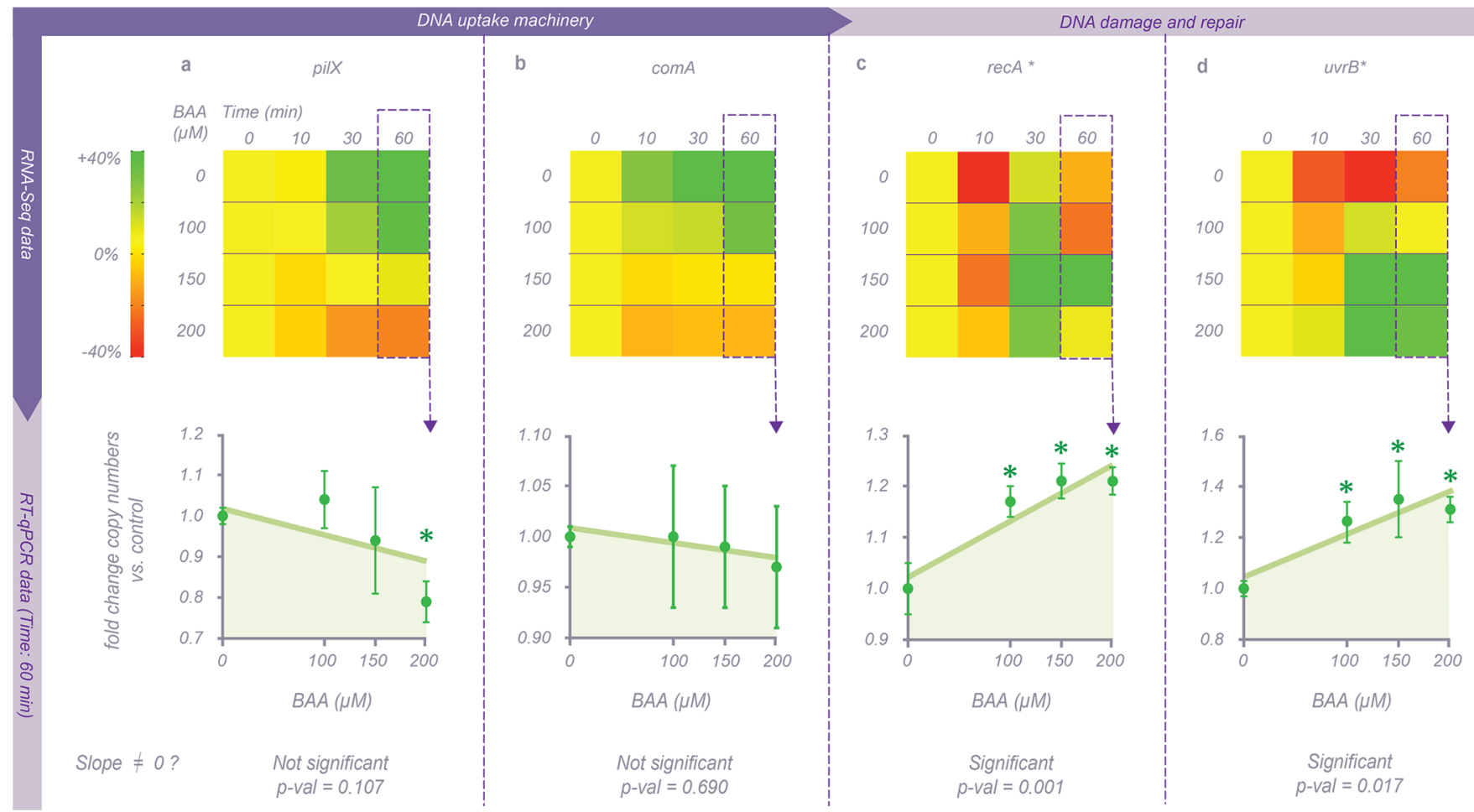

Figure 2. A. baylyi ADP1 transcriptional changes in response to BAA. The upper panel shows heat maps representing percent changes in transcript per million (TPM) abundance at $t_{i}$ vs $t=0$ min for genes associated with DNA translocation machinery, (a) pilX and (b) comA, and genes associated with DNA integration/repair, (c) recA and (d) $u v r B$. Lower panels correspond to samples at $t=60$ min quantified by RT-qPCR to confirm transcriptional changes observed by RNA-Seq Linear models fitted to fold changes in copy numbers to establish concentration-response transcriptional changes. Slopes of linear models were statically tested for deviation from zero using Graph Pad Prism 8 linear regression package with $\alpha=0.050$.

glutathione $(\mathrm{GSH})$ in the TA100 assay achieved a $73-91.4 \%$ reduction in the reversion frequency compared to that of the BAA treatment alone (Figure 1c). These results confirm the ability of GSH to modulate the ROS-mediated mutagenic response.

To further test the effect of GSH on the BAA stimulation of transformation rates in $A$. baylyi, we selected a concentration of $400 \mu \mathrm{M} \mathrm{BAA}$, as it exhibited a prominent transformation enhancement and cytotoxic response (Figure 1b). The addition of GSH completely reversed the increase of transformation rates by $400 \mu \mathrm{M}$ BAA in the reporter strain (Figure 1d) but did not have any effect on its cytotoxic response (Figure 1e). These data were statistically significant as compared to their concurrent controls and demonstrate a direct causation between ROSmediated DNA damage and the increase of transformation rates in BAA-treated assays.

The results of prior research with $A$. baylyi suggested that expression of natural transformation could lead to increased survival under stress conditions. ${ }^{45}$ Potentially, this could result in artifacts in the estimation of natural transformation rates in response to BAA, as increased survival of the transformants could lead to overestimation of transformation events in BAAtreated assays. However, this possibility could be ruled out on the basis of the fact that enhancement of transformation was also detected in the absence of cell death (i.e., noncytotoxic BAA levels, Figure $1 \mathrm{~b}$ ), suggesting that this response is not linked to cytotoxicity. This observation was further corroborated in the BAA + GSH coincubation assays. GSH showed no effect on the BAA cytotoxic response (Figure 1e); therefore, if the enhanced transformation phenotype was indeed an artifact resulting from increased survival of the transformants, it would be anticipated that the transformation rates observed for BAA and BAA + GSH be similar. Instead, the addition of GSH completely reversed the transformation frequencies in BAA coincubated treatments. These results provide strong evidence that the observed concentration-response enhancement of transformation frequencies was not the result of an artifact arising from increased survival to cytotoxic stress but rather was mediated by ROS.

3.3. A. baylyi Transcriptional Response to BAA Exposure. A toxico-genomic experiment was conducted to identify genes that were modulated by BAA concentrations (Figures S1 and S2, SI). Sample quality control data can be found in Table S3 (SI). Previous studies demonstrated that the monohalogenated HAAs, which include BAA, blocked glycolysis and reduced cellular ATP levels due to the inhibition of glyceraldehyde-3-phosphate dehydrogenase (GAPDH). ${ }^{44,46}$ Similarly, it was observed that in response to GAPDH inhibition and ROS formation, A. baylyi upregulated the expression of GAPDH and ROS detoxification response genes. Indeed, the transcriptomic data revealed a concentration-response increase in the transcripts per million (TPM) of both gene traits (Table S4 and Figure S4, SI) and provided a molecular mode of action for both the mutagenicity and enhanced transformation mediated by BAA concentration. These data were in agreement with toxico-genomic studies of BAA genotoxicity in human cell systems ${ }^{43,47-49}$ but were not yet reported for bacterial cell systems.

A comprehensive list of all genes following a concentrationresponse change in expression is shown in Table S5 (SI). To further elucidate how BAA would modulate gene expressions, genes related to natural transformation were emphasized. ${ }^{50}$ Natural transformation occurs via two steps, namely, DNA 

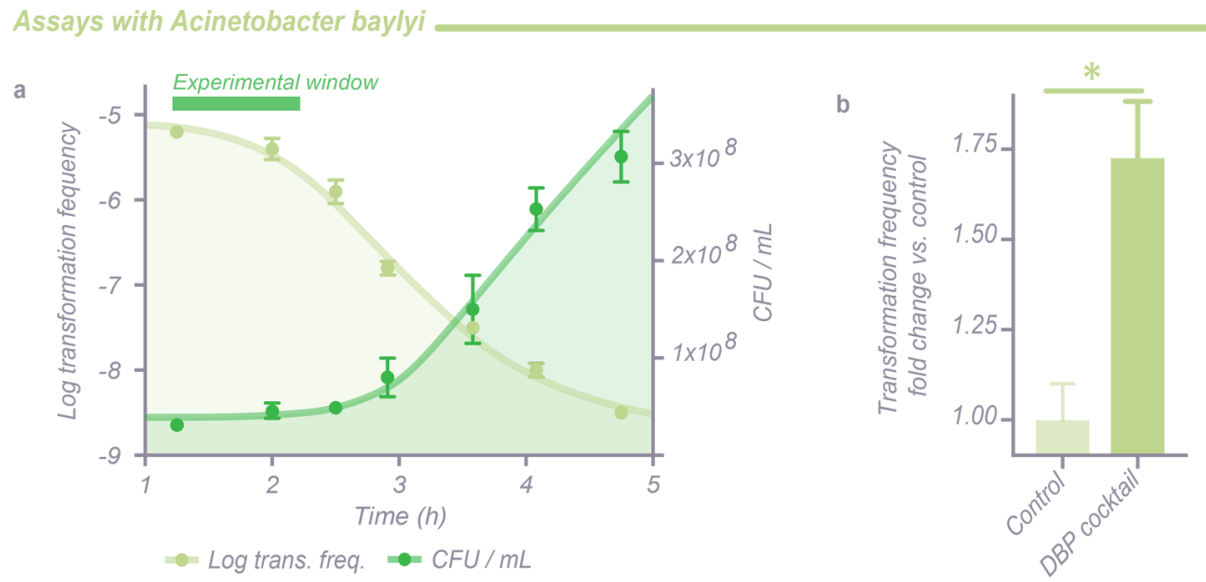

Figure 3. Environmental concentrations of DBPs stimulate transformation. (a) Growth-dependent donor DNA transformation frequencies in $A$. baylyi. Peak competence and transformation was detected at the very early stages of the growth curve, establishing the experimental window used for the DBP cocktail transformation assays. (b) Fold-change transformation frequencies of A. baylyi ADP1 exposed to a DBP cocktail simulating the environmental concentrations in treated wastewater effluents of five DBP classes (Table S2, SI) vs a mock-treated control $(n=3)$. ${ }^{\text {denotes } P<0.001 .}$

uptake and DNA integration. ${ }^{27}$ Two essential genes of the DNA uptake machinery, pilX and $\operatorname{com} A,{ }^{50}$ were observed to be downregulated with exposure to BAA by RNA-seq (Figure 2a,b). However, confirmation by RT-qPCR did not show a concentration-response pattern, since the slope of the foldchange trend line did not significantly differ from zero (Figure $2 a, b)$. Hence, the increase in natural transformation observed in response to BAA was unlikely due to an increase in active DNA uptake.

However, ROS generated by BAA may have altered the physical structure of cell membranes. The exact types of ROS generated by BAA are not known, but hydrogen peroxide is likely to be one of the generated ROS. This is inferred because the addition of glutathione (a scavenger for hydrogen peroxide ${ }^{51}$ ) was able to revert the mutagenicity level (Figure 1c) and restore natural transformation rates to that of the baseline level observed at $0 \mu \mathrm{M}$ BAA (Figure 1d). Hydrogen peroxide can accumulate in large amounts in the cell membranes, resulting in an increased oxidized lipid fraction, which would cause higher membrane permeability. ${ }^{52}$ This may result in a higher pool of donor DNA within the cytoplasm, but further studies would have to be conducted to establish if ROS would indeed result in higher DNA permeability.

Next, genes related to DNA integration were further examined. ROS-induced DNA damage causes primarily singlestrand breaks (SSB), while double-strand breaks occur at lower frequencies as a consequence of two proximal SSB or following replication passing through ROS-induced lesions. ${ }^{53}$ In toxicogenomic studies, BAA exposure modulated the expression of genes involved in both SSB and DNA double-strand break repair. ${ }^{47}$ While SSB are repaired by nucleotide excision, doublestrand breaks are primarily repaired via homologous recombination in a RecA-dependent manner. ${ }^{26}$

As RecA is the molecular link between DNA repair and natural transformation in our hypothesis, we expected overexpression of the $r e c A$ gene in response to BAA treatment. In accordance to this hypothesis, transcripts for $\operatorname{rec} A$ were more abundant in BAA treatments than in controls, however, contrary to other SOS-controlled genes such as himA ${ }^{54}$ (Table S4, SI); the $\operatorname{rec} A$ induction profile did not follow a clear BAA concentration-response behavior at $t=60 \mathrm{~min}$ (Figure 2c). To exemplify, recA exhibited more downregulation in the absence of BAA than with $100-200 \mu \mathrm{M} \mathrm{BAA}$ at $t=10 \mathrm{~min}$. The gene then experienced an increase in TMP abundance at $30 \mathrm{~min}$, and the upregulation was higher in the presence of BAA than in the control. There was however an outlier trend at $t=60 \mathrm{~min}$ (Figure 2c). RT-qPCR was used to further verify RNA-Seq data at this time point and revealed a clear linear increase in $r e c A$ copy numbers in response to BAA at $t=60 \mathrm{~min}$. We cannot explain the discrepancy between the results derived from RNA-Seq and $\mathrm{RT}$-qPCR at $t=60 \mathrm{~min}$; however, RNA-Seq is a global gene expression profiling tool and may not exactly reflect the expression status of all genes. On the other hand, RT-qPCR is a highly targeted approach, which makes single gene expression data less sensitive to background variations.

Other components of the DNA repair machinery, such as $u v r B$, an essential constituent of the nucleotide excision and repair complex, was transcribed in a BAA concentrationdependent manner in both the RNA-Seq and RT-qPCR data (Figure $2 \mathrm{~d}$ ). These results are consistent with previous studies describing the mutagenic mode of action of $\mathrm{BAA},{ }^{47}$ indicating that DNA damage response to the BAA-induced mutagenicity was predominantly SSB repair.

When examining the expression patterns of genes associated with natural transformation, only $\operatorname{rec} A$ showed consistent transcriptional modulation in response to BAA in both RNASeq and RT-qPCR data sets (Figure 2c). The participation of RecA in DNA repair and DNA integration during natural transformation led us to consider $r e c A$ overexpression as the mechanism potentially responsible for the increase of natural transformation rates.

3.4. Environmental Concentrations of DBPs Increase Natural Transformation Rates. A DBP cocktail that contained environmental concentrations of five classes of DBPs, including those regulated by the U.S. Environmental Protection Agency ${ }^{55}$ (Table S2, SI), was further used to test the hypothesis that enhanced transformation would be associated with DBPs. A. baylyi cell cultures were exposed to the DBP cocktail during early exponential phase as we found that, at this point, A. baylyi cell cultures expressed peak transformation frequencies (Figure 3a). Under these conditions, the DBP mixture stimulated donor DNA transformation by nearly 2 -fold (Figure $3 \mathrm{~b}$ ). These results demonstrate the environmental relevance of the phenomenon herein described in this study. 


\section{IMPLICATIONS}

This study reveals that water disinfection byproducts significantly increase the transformation rates of extracellular DNA in the bacterial model A. baylyi ADP1. The stimulation phenotype is linked to the ability of BAA to cause DNA damage via oxidative stress, which triggered increased transcription of $r e c A$, a key recombinase involved in both DNA damage/repair and foreign DNA integration. The higher cytoplasmic levels of RecA could subsequently increase the chances of foreign DNA to be integrated in the bacterial chromosome and hence resulted in an increase in natural transformation. It is important to highlight that DNA damage is the ultimate factor driving the stimulation of transformation, and therefore, exposure to other environmental conditions (e.g., chlorination per se, solar irradiance, heavy metals) that generate mutagenic damage could potentially result in a similar and/or synergic stimulation response.

Given that chlorine is a common disinfection strategy in most wastewater treatment plants and that the formation of DBPs is an inevitable process, the implications of DBP-enhanced natural transformation rates are broad. Previous studies have shown that DBPs and other chemical pollutants present in wastewater increase conjugation rates among diverse lineages of bacteria. $^{56-59}$ However, to the best of our knowledge, the effect of DBPs over other mechanisms of HGT (i.e., natural transformation) has not been described yet. Unlike conjugation, natural transformation allows for the exchange of information among phylogenetically more distant microorganisms. ${ }^{27}$ Therefore, environmental compartments with mutagenic potentialsuch as water bodies impacted by wastewater discharges-could facilitate the mobilization of novel ARGs from soil organisms to microorganisms of clinical relevance.

Even though conjugation and natural transformation are independent mechanisms of HGT, there appear to be several shared elements between the DBP-mediated enhancement of transformation and previous studies on stimulation of conjugation by disinfectants and other environmental pollutants. ${ }^{57,59}$ These elements include (i) the generation of ROS and (ii) the subsequent activation of stress response. While previous studies showed a correlation between the intracellular ROS production and increased conjugation rates, here we established a direct link between ROS-mediated DNA damage and the enhancement of transformation. Although $A$. baylyi does not possess a prototypical S.O.S response, ${ }^{60}$ in agreement with previous research, ${ }^{57,59}$ we were able to detect downstream activation of stress-response pathways, as evidenced by the increased transcription of ROS detoxification and DNA repair genes (i.e., $\operatorname{rec} A$ and $u v r B$ ).

While conjugation might be the predominant factor influencing the environmental spread of ARGs, we speculate that the volumetric magnitude of wastewater discharges, along with the DBPs, could significantly contribute to the persistence of ARGs that exist as naked extracellular DNA. In our opinion, the results from this work help us to elaborate a more comprehensive view of ARG dissemination in the environmental metagenome, introducing extra layers of information concerning (i) the ultimate fate of naked extracellular DNA, as well as (ii) improved genetic exchange beyond the host range of mobile genetic elements and bacteriophages. ${ }^{27}$ Collectively, these findings raise concerns over our current reliance on conventional chlorine-based wastewater disinfection and suggest that disinfection strategies should be evaluated not only based on their inactivation efficiencies but also their potential to stimulate horizontal gene transfer.

\section{ASSOCIATED CONTENT}

\section{Supporting Information}

The Supporting Information is available free of charge on the ACS Publications website at DOI: 10.1021/acs.est.9b00692.

Methods for biomass collection for BAA toxico-genomics and RNA extraction; tables summarizing primers for RTqPCR, composition of the simplified disinfection byproduct cocktail, quality control of transcriptome samples, selected differentially expressed genes following a dose-response behavior relative to BAA concentration (Tables S1-S4); and figures showing BAA exposure time selection and BAA cytotoxicity for toxico-genomic experiment, toxico-genomic experimental design, and verification of transcriptomic data using housekeeping gene with known response to BAA (Figures S1-S4) (PDF)

Comprehensive list of differently expressed genes following a dose-response behavior relative to BAA concentration (Table S5) (XLSX)

\section{AUTHOR INFORMATION}

\section{Corresponding Author}

*E-mail: peiying.hong@kaust.edu.sa; phone: +966-12-8082218. ORCID

Michael J. Plewa: 0000-0001-8307-1629

Pei-Ying Hong: 0000-0002-4474-6600

\section{Notes}

The authors declare no competing financial interest.

All transcriptomic FASTQ files are deposited in the European Nucleotide Archive under study accession number PRJEB28698.

\section{ACKNOWLEDGMENTS}

This study is supported by KAUST baseline funding BAS/1/ 1033-01-01 awarded to P.-Y.H.

\section{REFERENCES}

(1) Laxminarayan, R.; Duse, A.; Wattal, C.; Zaidi, A. K.; Wertheim, H. F.; Sumpradit, N.; Vlieghe, E.; Hara, G. L.; Gould, I. M.; Goossens, H.; et al. Antibiotic resistance-the need for global solutions. Lancet Infect. Dis. 2013, 13 (12), 1057-1098.

(2) Forsberg, K. J.; Reyes, A.; Wang, B.; Selleck, E. M.; Sommer, M. O.; Dantas, G. The shared antibiotic resistome of soil bacteria and human pathogens. Science 2012, 337 (6098), 1107-1111.

(3) Czekalski, N.; Gascon Diez, E.; Burgmann, H. Wastewater as a point source of antibiotic-resistance genes in the sediment of a freshwater lake. ISME J. 2014, 8 (7), 1381-90.

(4) Ahammad, Z. S.; Sreekrishnan, T. R.; Hands, C. L.; Knapp, C. W.; Graham, D. W. Increased waterborne blaNDM-1 resistance gene abundances associated with seasonal human pilgrimages to the upper ganges river. Environ. Sci. Technol. 2014, 48 (5), 3014-20.

(5) Luo, Y.; Yang, F.; Mathieu, J.; Mao, D.; Wang, Q.; Alvarez, P. J. J. Proliferation of Multidrug-Resistant New Delhi Metallo- $\beta$-lactamase Genes in Municipal Wastewater Treatment Plants in Northern China. Environ. Sci. Technol. Lett. 2014, 1 (1), 26-30.

(6) Mantilla-Calderon, D.; Jumat, M. R.; Wang, T.; Ganesan, P.; AlJassim, N.; Hong, P.-Y. Isolation and characterization of NDM-positive Escherichia coli from municipal wastewater in Jeddah, Saudi Arabia. Antimicrob. Agents Chemother. 2016, 60 (9), 5223-5231. 
(7) Wellington, E. M.; Boxall, A. B.; Cross, P.; Feil, E. J.; Gaze, W. H.; Hawkey, P. M.; Johnson-Rollings, A. S.; Jones, D. L.; Lee, N. M.; Otten, $\mathrm{W}$.; et al. The role of the natural environment in the emergence of antibiotic resistance in Gram-negative bacteria. Lancet Infect. Dis. 2013, 13 (2), 155-165.

(8) Mao, D.; Yu, S.; Rysz, M.; Luo, Y.; Yang, F.; Li, F.; Hou, J.; Mu, Q.; Alvarez, P. J. Prevalence and proliferation of antibiotic resistance genes in two municipal wastewater treatment plants. Water Res. 2015, 85, $458-66$.

(9) Al-Jassim, N.; Ansari, M. I.; Harb, M.; Hong, P. Y. Removal of bacterial contaminants and antibiotic resistance genes by conventional wastewater treatment processes in Saudi Arabia: Is the treated wastewater safe to reuse for agricultural irrigation? Water Res. 2015, 73, 277-90.

(10) Massalha, N.; Dong, S.; Plewa, M. J.; Borisover, M.; Nguyen, T. $\mathrm{H}$. Spectroscopic indicators for cytotoxicity of chlorinated and ozonated effluents from wastewater stabilization ponds and activated sludge. Environ. Sci. Technol. 2018, 52, 3167-3174.

(11) Dong, S.; Massalha, N.; Plewa, M. J.; Nguyen, T. H. The impact of disinfection $\mathrm{Ct}$ values on cytotoxicity of agricultural wastewaters: ozonation vs. chlorination. Water Res. 2018, 144, 482-490.

(12) Dong, S.; Masalha, N.; Plewa, M. J.; Nguyen, T. H. Toxicity of wastewater with elevated bromide and iodide after chlorination, chloramination, or ozonation disinfection. Environ. Sci. Technol. 2017, 51, 9297-9304.

(13) Dong, S.; Nguyen, T. H.; Plewa, M. J. Comparative mammalian cell cytotoxicity of wastewater with elevated bromide and iodide after chlorination, chloramination, or ozonation. J. Environ. Sci. 2017, 58, 296-301.

(14) Dong, S.; Lu, J.; Plewa, M. J.; Nguyen, T. H. Comparative mammalian cell cytotoxicity of wastewaters for agricultural reuse after ozonation or chlorination. Environ. Sci. Technol. 2016, 50, 1175211759.

(15) Guardabassi, L.; Petersen, A.; Olsen, J. E.; Dalsgaard, A. Antibiotic resistance in Acinetobacter spp. isolated from sewers receiving waste effluent from a hospital and a pharmaceutical plant. Appl. Environ. Microbiol. 1998, 64 (9), 3499-3502.

(16) Zhang, Y.; Marrs, C. F.; Simon, C.; Xi, C. Wastewater treatment contributes to selective increase of antibiotic resistance among Acinetobacter spp. Sci. Total Environ. 2009, 407 (12), 3702-3706.

(17) Goñi-Urriza, M.; Capdepuy, M.; Arpin, C.; Raymond, N.; Caumette, P.; Quentin, C. Impact of an Urban Effluent on Antibiotic Resistance of Riverine Enterobacteriaceae andAeromonas spp. Applied and environmental Microbiology. 2000, 66 (1), 125-132.

(18) Rizzo, L.; Manaia, C.; Merlin, C.; Schwartz, T.; Dagot, C.; Ploy, M. C.; Michael, I.; Fatta-Kassinos, D. Urban wastewater treatment plants as hotspots for antibiotic resistant bacteria and genes spread into the environment: A review. Sci. Total Environ. 2013, 447, 345-360.

(19) Li, A.-D.; Li, L.-G.; Zhang, T. Exploring antibiotic resistance genes and metal resistance genes in plasmid metagenomes from wastewater treatment plants. Front. Microbiol. 2015, 6, 1025.

(20) Guo, M.-T.; Yuan, Q.-B.; Yang, J. Distinguishing effects of ultraviolet exposure and chlorination on the horizontal transfer of antibiotic resistance genes in municipal wastewater. Environ. Sci. Technol. 2015, 49 (9), 5771-5778.

(21) Von Wintersdorff, C. J.; Penders, J.; Van Niekerk, J. M.; Mills, N. D.; Majumder, S.; Van Alphen, L. B.; Savelkoul, P. H.; Wolffs, P. F. Dissemination of antimicrobial resistance in microbial ecosystems through horizontal gene transfer. Front. Microbiol. 2016, 7, 173.

(22) Richardson, S. D.; Plewa, M. J.; Wagner, E. D.; Schoeny, R.; Demarini, D. M. Occurrence, genotoxicity, and carcinogenicity of regulated and emerging disinfection by-products in drinking water: a review and roadmap for research. Mutat. Res., Rev. Mutat. Res. 2007, 636 (1-3), 178-242.

(23) Wagner, E. D.; Plewa, M. J. CHO cell cytotoxicity and genotoxicity analyses of disinfection by-products: an updated review. J. Environ. Sci. 2017, 58, 64-76.

(24) Min, J.; Kim, E. J.; LaRossa, R. A.; Gu, M. B. Distinct responses of a recA::luxCDABE Escherichia coli strain to direct and indirect DNA damaging agents. Mutat. Res., Genet. Toxicol. Environ. Mutagen. 1999, $442(2), 61-68$.

(25) Rauch, P. J.; Palmen, R.; Burds, A. A.; Gregg-Jolly, L. A.; Van der Zee, J. R.; Hellingwerf, K. J. The expression of the Acinetobacter calcoaceticus recA gene increases in response to DNA damage independently of RecA and of development of competence for natural transformation. Microbiology 1996, 142 (4), 1025-1032.

(26) Hiom, K. DNA repair: common approaches to fixing doublestrand breaks. Curr. Biol. 2009, 19 (13), R523-R525.

(27) Thomas, C. M.; Nielsen, K. M. Mechanisms of, and barriers to, horizontal gene transfer between bacteria. Nat. Rev. Microbiol. 2005, 3 (9), 711.

(28) Krasner, S. W.; Weinberg, H. S.; Richardson, S. D.; Pastor, S. J.; Chinn, R.; Sclimenti, M. J.; Onstad, G. D.; Thruston, A. D. Occurrence of a new generation of disinfection byproducts. Environ. Sci. Technol. 2006, 40 (23), 7175-7185.

(29) Zeng, T.; Plewa, M. J.; Mitch, W. A. Chemical and toxicological assessment of $\mathrm{N}$-nitrosamines and halogenated disinfection byproducts in potable reuse treatment trains. Water Res. 2016, 101, 176-186.

(30) Kargalioglu, Y.; McMillan, B. J.; Minear, R. A.; Plewa, M. J. Analysis of the cytotoxicity and mutagenicity of drinking water disinfection by-products in Salmonella typhimurium. Teratog., Carcinog., Mutagen. 2002, 22 (2), 113-128.

(31) Plewa, M. J.; Simmons, J. E.; Richardson, S. D.; Wagner, E. D. Mammalian cell cytotoxicity and genotoxicity of the haloacetic acids, a major class of drinking water disinfection by-products. Environ. Mol. Mutagen. 2010, 51, 871-878.

(32) Rizzi, A.; Pontiroli, A.; Brusetti, L.; Borin, S.; Sorlini, C.; Abruzzese, A.; Sacchi, G. A.; Vogel, T. M.; Simonet, P.; Bazzicalupo, M.; Nielsen, K. M.; Monier, J. M.; Daffonchio, D. Strategy for in situ detection of natural transformation-based horizontal gene transfer events. Appl. Environ. Microbiol. 2008, 74 (4), 1250-4.

(33) Maron, D. M.; Ames, B. N. Revised methods for the Salmonella mutagenicity test. Mutation Research/Environmental Mutagenesis and Related Subjects. 1983, 113 (3-4), 173-215.

(34) Hengstler, J. G.; Oesch, F. In Encyclopedia of Gentics; Academic Press, 2001; pp 51-54.

(35) Andrews, S. FastQC: A quality control tool for high throughput sequence data 2014. http://www.bioinformatics.babraham.ac.uk/ projects/fastqc/.

(36) Bolger, A. M.; Lohse, M.; Usadel, B. Trimmomatic: a flexible trimmer for Illumina sequence data. Bioinformatics 2014, 30 (15), 2114-2120.

(37) Bray, N. L.; Pimentel, H.; Melsted, P.; Pachter, L. Near-optimal probabilistic RNA-seq quantification. Nat. Biotechnol. 2016, 34 (5), $525-527$

(38) Pimentel, H.; Bray, N. L.; Puente, S.; Melsted, P.; Pachter, L. Differential analysis of RNA-seq incorporating quantification uncertainty. Nat. Methods 2017, 14 (7), 687-690.

(39) Al-Jassim, N.; Mantilla-Calderon, D.; Wang, T.; Hong, P. Inactivation of a virulent wastewater Escherichia coli and non-virulent commensal Escherichia coli DSM1103 strains and their gene expression upon solar irradiation. Environ. Sci. Technol. 2017, 51 (7), 3649-3659.

(40) Maron, D. M.; Ames, B. N. Revised methods for the Salmonella mutagenicity test. Mutat. Res. 1983, 113 (3-4), 173-215.

(41) Pals, J. A.; Wagner, E. D.; Plewa, M. J. Energy of the lowest unoccupied molecular orbital, thiol reactivity, and toxicity of three monobrominated water disinfection byproducts. Environ. Sci. Technol. 2016, 50 (6), 3215-3221.

(42) Cemeli, E.; Wagner, E. D.; Anderson, D.; Richardson, S. D.; Plewa, M. J. Modulation of the cytotoxicity and genotoxicity of the drinking water disinfection byproduct iodoacetic acid by suppressors of oxidative stress. Environ. Sci. Technol. 2006, 40 (6), 1878-1883.

(43) Pals, J.; Attene-Ramos, M. S.; Xia, M.; Wagner, E. D.; Plewa, M. J. Human cell toxicogenomic analysis linking reactive oxygen species to the toxicity of monohaloacetic acid drinking water disinfection byproducts. Environ. Sci. Technol. 2013, 47 (21), 12514-12523.

(44) Pals, J. A.; Ang, J. K.; Wagner, E. D.; Plewa, M. J. Biological mechanism for the toxicity of haloacetic acid drinking water 
disinfection byproducts. Environ. Sci. Technol. 2011, 45 (13), 57915797.

(45) Hülter, N.; Sørum, V.; Borch-Pedersen, K.; Liljegren, M. M.; Utnes, A. L.; Primicerio, R.; Harms, K.; Johnsen, P. J. Costs and benefits of natural transformation in Acinetobacter baylyi. BMC Microbiol. 2017, 17 (1), 34.

(46) Dad, A.; Jeong, C. H.; Pals, J.; Wagner, E. D.; Plewa, M. J. Pyruvate remediation of cell stress and genotoxicity induced by haloacetic acid drinking water disinfection byproducts. Environ. Mol. Mutagen. 2013, 54, 629-637.

(47) Muellner, M. G.; Attene-Ramos, M. S.; Hudson, M. E.; Wagner, E. D.; Plewa, M. J. Human cell toxicogenomic analysis of bromoacetic acid: a regulated drinking water disinfection by-product. Environ. Mol. Mutagen. 2009, 51, 205-214.

(48) Pals, J. A.; Wagner, E. D.; Plewa, M. J.; Xia, M.; Attene-Ramos, M. $\mathrm{S}$. Monohalogenated acetamide-induced cellular stress and genotoxicity are related to electrophilic softness and thiol/thiolate reactivity. $J$. Environ. Sci. 2017, 58, 224-230.

(49) Attene-Ramos, M. S.; Wagner, E. D.; Plewa, M. J. Comparative human cell toxicogenomic analysis of monohaloacetic acid drinking water disinfection byproducts. Environ. Sci. Technol. 2010, 44 (19), $7206-7212$.

(50) Leong, C. G.; Bloomfield, R. A.; Boyd, C. A.; Dornbusch, A. J.; Lieber, L.; Liu, F.; Owen, A.; Slay, E.; Lang, K. M.; Lostroh, C. P. The role of core and accessory type IV pilus genes in natural transformation and twitching motility in the bacterium Acinetobacter baylyi. PLoS One 2017, 12 (8), No. e0182139.

(51) Mishra, S.; Imlay, J. Why do bacteria use so many enzymes to scavenge hydrogen peroxide? Arch. Biochem. Biophys. 2012, 525 (2), $145-160$.

(52) Itri, R.; Junqueira, H. C.; Mertins, O.; Baptista, M. S. Membrane changes under oxidative stress: the impact of oxidized lipids. Biophys. Rev. 2014, 6 (1), 47-61.

(53) Lindahl, T. Instability and decay of the primary structure of DNA. Nature 1993, 362 (6422), 709-715.

(54) Miller, H. I.; Kirk, M.; Echols, H. SOS induction and autoregulation of the himA gene for site-specific recombination in Escherichia coli. Proc. Natl. Acad. Sci. U. S. A. 1981, 78 (11), 67546758.

(55) U.S. Environmental Protection Agency.. National primary drinking water regulations: Stage 2 disinfectants and disinfection byproducts rule. Fed. Regist. 2006, 71 (2), 387-493.

(56) Li, D.; Zeng, S.; He, M.; Gu, A. Z. Water disinfection byproducts induce antibiotic resistance-role of environmental pollutants in resistance phenomena. Environ. Sci. Technol. 2016, 50 (6), 3193-3201.

(57) Zhang, Y.; Gu, A. Z.; He, M.; Li, D.; Chen, J. Sub-inhibitory Concentrations of Disinfectants Promote the Horizontal Transfer of Multidrug Resistance Genes within and across Genera. Environ. Sci. Technol. 2017, 51 (1), 570-580.

(58) Lu, J.; Jin, M.; Nguyen, S. H.; Mao, L.; Li, J.; Coin, L. J.; Yuan, Z.; Guo, J. Non-antibiotic antimicrobial triclosan induces multiple antibiotic resistance through genetic mutation. Environ. Int. 2018, $118,257-265$.

(59) Wang, Y.; Lu, J.; Mao, L.; Li, J.; Yuan, Z.; Bond, P. L.; Guo, J. Antiepileptic drug carbamazepine promotes horizontal transfer of plasmid-borne multi-antibiotic resistance genes within and across bacterial genera. ISME J. 2019, 13 (2), 509-522.

(60) Hare, J. M.; Ferrell, J. C.; Witkowski, T. A.; Grice, A. N. Prophage induction and differential RecA and UmuDAb transcriptome regulation in the DNA damage responses of Acinetobacter baumannii and Acinetobacter baylyi. PLoS One 2014, 9 (4), No. e93861. 